\title{
Metastatic Cardiac Tumor Manifested by Persistent ST-Segment Elevation With Coexisting Reciprocal Changes on Electrocardiography
}

\author{
Dae Young Cheon, ${ }^{1}$ MD, Kyoung-Ha PARK, ${ }^{1}$ MD, Seong Eun Hong, ${ }^{1}$ MD, \\ Soo Haeng LeE, ${ }^{1}$ MD, Seung Hun JANG, ${ }^{2}$ MD, and Woo Jung PArK, ${ }^{1}$ MD
}

\begin{abstract}
SUMMARY
In cases with metastatic invasion of the heart, electrocardiographic abnormalities are commonly seen. However, most of these electrocardiographic changes are nonspecific; certain findings may be highly suggestive of myocardial involvement of the tumor. We report a patient with lung cancer who presented with persistent ST-segment elevation with coexisting reciprocal changes on electrocardiography due to myocardial invasion of the lung cancer. (Int Heart J 2014; 55: 466-468)
\end{abstract}

Key words: Cardiac metastasis, ST-segment change

$\mathrm{A}$ lthough acute myocardial infarction is one of the most frequent causes of ST-segment elevation, there are other reasons for electrocardiographic changes, such as invasion of the metastatic cancer on the myocardium. ${ }^{1)}$ In the present report, we describe the case of a patient with nonsmall cell lung carcinoma (NSCLC) who presented with persistent ST-segment elevation with coexisting reciprocal changes on electrocardiography (ECG).

\section{Case Report}

A 70-year-old man was referred to our hospital due to pain in the left upper chest wall. A 12-lead ECG was normal and chest X-rays showed patchy consolidation in the left upper lobe. Chest computed tomography (CT) revealed a $4.3-\mathrm{cm}$ lung mass with adjacent chest wall invasion, and NSCLC was diagnosed by percutaneous needle biopsy. The clinical stage of NSCLC was T3N1Mx, and palliative radiation therapy and chemotherapy were administered to the patient. However, the follow-up CT showed progression of the lung cancer. Five months later, the patient was admitted due to generalized weakness. The patient's blood pressure was $122 / 70 \mathrm{mmHg}$ and the heart rate was 89 beats per minute. The ECG showed a sinus rhythm with ST-segment elevation in the inferior leads of II, III, and aVF with reciprocal ST-segment depression in the antero-lateral leads of aVL and V1-V4 (Figure 1A). However, the patient had no chest pain, and the cardiac markers were within the normal ranges: creatine kinase $78 \mathrm{IU} / \mathrm{L}$ (22 269 IU/ L), CK-MB $2.7 \mathrm{ng} / \mathrm{mL}(0 \sim 7.2 \mathrm{ng} / \mathrm{mL})$, and troponin-I $0.1 \mathrm{ng} /$ $\mathrm{mL}(0 \sim 0.3 \mathrm{ng} / \mathrm{mL})$. Transthoracic echocardiography revealed irregularly and severely thickened (about $2.5 \mathrm{~cm}$ ) inferior and posterior-lateral walls of the left ventricle, which suggested tumor invasion of the myocardium and pericardium (Figure 2A and 2B). Although the thickened left ventricular wall showed diffuse hypokinesia, there were no definitive findings related to myocardial ischemia. Coronary angiography was not considered due to the findings of the echocardiography and clinical situation of the patient. The serial cardiac enzymes at 6 hours and 24 hours later and follow-up echocardiography showed no gross interval changes compared with previous results. These findings helped us to exclude the presence of myocardial infarction. The chest CT and positron emission tomographycomputed tomography (PET-CT) findings revealed mass invasion of the inferior and posterolateral wall of the left ventricle (Figure 3A and 3B). In addition, the PET-CT showed intense fluorodeoxyglucose (FDG) uptake in the left perihilar and hilar areas and multiple metastases in bones and muscles of the lumbosacral area and right shoulder, which supported lymphatogenous or hematogenous metastasis of the lung cancer in the left ventricle. The patient was managed with supportive care and palliative chemotherapy. Two months later, the patient was relatively stable and had no symptoms of myocardial ischemia. However, a follow-up CT showed an increased size of the lung cancer and aggravation of the multiple bone and soft tissue metastases. Although there was scanty pericardial effusion, the metastatic mass of the left ventricle was aggravated. The follow-up ECG showed progression of the ST-segment elevation in inferior leads (about $0.1 \mathrm{mV}$ ) and deepening of the coexisting reciprocal ST-segment depression on antero-lateral leads (about $0.2 \mathrm{mV}$ ) compared with two months previously (Figure 1B). Three-months later, his clinical condition deteriorated due to progression of lung cancer with severe pneumonia, and he was transferred to the local hospice for terminal

From the Divisions of ${ }^{1}$ Cardiology and ${ }^{2}$ Pulmonology, Department of Internal Medicine, Hallym University Medical Center, Anyang, Korea

Address for correspondence: Kyoung-Ha Park, MD, Division of Cardiovascular, Department of Internal Medicine, Hallym University Medical Center, 896, Pyeongchon-dong, Dongan-gu, Anyang-si, Gyeonggi-do 431-070, Korea. E-mail: pkhmd@naver.com

Received for publication February 18, 2014. Revised and accepted March 26, 2014

Released in advance online on J-STAGE August 4, 2014.

All rights reserved by the International Heart Journal Association. 
(A)

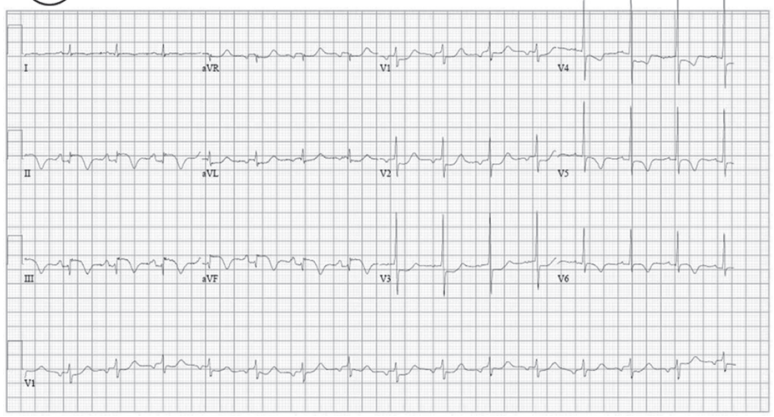

(B)



Figure 1. Changes in electrocardiography. An ECG showed ST-segment elevation in the inferior leads of II, III, and aVF with coexisting reciprocal STsegment depression in the antero-lateral leads of aVL and V1-V4 (A). Two months later, there was progression of the ST-segment elevation in inferior leads (about $0.1 \mathrm{mV}$ ) and deepening of the reciprocal ST-segment depression in antero-lateral leads (about $0.2 \mathrm{mV}$ ) on ECG (B).


Figure 2. Echocardiography findings. Transthoracic apical two-chamber (A) and apical three-chamber (B) views of the left ventricle, showing a large tumor mass invading the inferior and posterolateral walls of the myocardium and pericardium (white arrows).
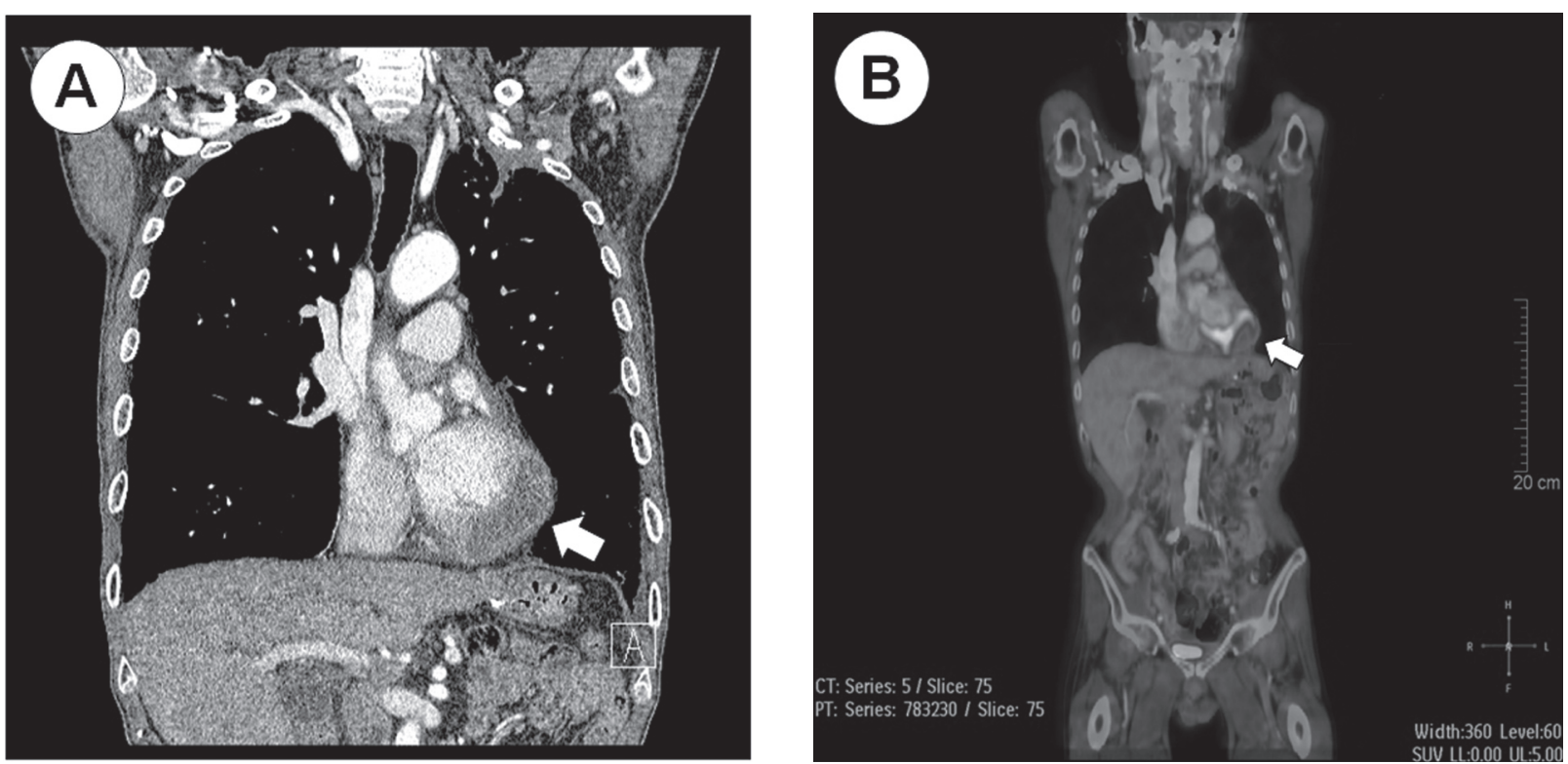

Figure 3. Findings of the chest computed tomography, and positron emission tomography-computed tomography. Chest computed tomography showed thickening of the inferior and posterolateral walls of the left ventricle (A, white arrow) and positron emission tomography-computed tomography showed intense fluorodeoxyglucose uptake in the pericardium and myocardium (B, white arrow), consistent with left ventricular invasion of the metastatic mass. 
care.

\section{DiscuSSION}

Metastatic cancer in the heart is nonspecific and clinically silent and myocardial metastasis rarely imitates myocardial infarction. ${ }^{2)}$ Secondary cardiac involvement by metastatic cancer is relatively uncommon and usually occurs when the disease is highly disseminated. ${ }^{3)}$

ECG changes in cardiac metastasis are not specific for tumor involvement and are difficult to differentiate from those associated with pre-existing heart disease or cardiotoxicity from cancer chemotherapy. ${ }^{4}$ There have been multiple isolated reports of ECG abnormalities associated with metastatic cancer involving the heart: (a) myocardial changes; segmental or diffuse T wave inversion, and ST-segment elevation, (b) conduction system changes; bundle branch block, fascicular block, atrioventricular block, atrial fibrillation, arterial flutter, and paroxysmal arterial tachycardia, (c) pericardial changes; ST-segment elevation and electrical alternans, (d) nonspecific changes; sinus tachycardia, nonspecific ST and T-wave abnormalities, low voltage, premature contractions, and (e) multiple combined abnormal ECG patterns. ${ }^{4-9)}$ In this case, the ECG showed persistent ST-segment elevation in inferior leads with coexisting reciprocal ST-segment depression in anterolateral leads without symptoms of myocardial ischemia. In addition, there was no arrhythmia such as supraventricular tachycardia or ventricular tachycardia.

In terms of the ST-segment changes, Cates, et al reported that among the patients who were diagnosed with cardiac metastasis on autopsy, a few patients showed ST-segment elevation on ECG. ${ }^{4)}$ The mechanism of persistent ST-segment elevation in tumor invasion of the heart is unknown. Hartman, et al suggested the following possible mechanisms: (a) the development of an injury that is current secondary to an inflammatory reaction around the tumor, (b) the ionic transfer of potassium from necrotic tissue to the adjacent myocardium, producing electropotential differences between these areas, and (c) the stretch of adjacent muscle fibers that lead to an injury current produced by the same mechanism of ST-segment elevation as is seen in a left ventricular aneurysm. ${ }^{4)}$ In this case, there was persistent ST-segment elevation in inferior leads due to cardiac metastasis of the lung cancer. Moreover, persistent reciprocal ST-segment depression was coexisting in antero-lateral leads, which differentiated our case from previous reports. Pericardial disease or aneurysmal changes of the left ventricle could be excluded because of the coexisting ST-segment depression in our case. The possible mechanisms of the persistent ST-segment depression on precordial leads are (a) pure electrical phenomenon with ST-segment elevation, (b) constant compression of the coronary arteries with resulting ischemia, and (c) com- bined coronary artery disease. It is hard to discriminate the precise cause of the persistent ST-segment depression; pure electrical phenomenon was the most plausible mechanism in this case because there was no evidence of myocardial invasion of the metastatic mass in the territory of the left anterior descending artery and the patient was not suffering from anginal pain. Samaras, et al reported more pronounced ST-segment elevations in relation to the growth of the myocardial metastasis of primary lung cancer. ${ }^{10)}$ In this case, there was not only progression of the ST-segment elevation (about $0.1 \mathrm{mV}$ ) but also deepening of the coexisting reciprocal ST-segment depression (about $0.2 \mathrm{mV}$ ) with the worsening of the myocardial metastasis. By including ST-segment elevation with coexisting reciprocal changes mimicking acute myocardial infarction, any new ECG changes should raise the suspicion of cardiac metastasis and lead to proper work-up in the management of patients with cancer.

Conclusions: In the absence of clinical symptoms of angina in patients with malignancy, any new ECG changes suggestive of myocardial ischemia should lead investigators to exclude cardiac metastasis.

\section{REFERENCES}

1. Harris TR, Copeland GD, Brody DA. Progressive injury current with metastatic tumor of the heart: case report and review of the literature. Am Heart J 1965; 69: 392-400.

2. Pan KL, Wu LS, Chung CM, Chang ST, Lin PC, Hsu JT. Misdiagnosis: cardiac metastasis presented as a pseudo-infarction on electrocardiography. Int Heart J 2007; 48: 399-405.

3. Vallot F, Berghmans T, Delhaye F, Dagnelie J, Sculier JP. Electrocardiographic manifestations of heart metastasis from a primary lung cancer. Support Care Cancer 2001; 9: 275-7.

4. Hartman RB, Clark PI, Schulman P. Pronounced and prolonged ST segment elevation: a pathognomonic sign of tumor invasion of the heart. Arch Intern Med 1982; 142: 1917-9.

5. Zatuchni J, Burris A, Vejviboonsom P, Voci G. Metastatic epidermoid cardiac tumor manifested by persistent ST segment elevation. Am Heart J 1981; 101: 674-5.

6. Koiwaya Y, Nakamura M, Yamamoto K. Progressive ECG alterations in metastatic cardiac mural tumor. Am Heart J 1983; 105: 339-41.

7. Htoo MM, Nanton MA. Complete heart block due to disseminated vulval carcinoma. Br Heart J 1973; 35: 1211-3.

8. Cates CU, Virmani R, Vaughn WK, Robertson RM. Electrocardiographic markers of cardiac metastasis. Am Heart J 1986; 112: 1297-303.

9. James TN, Carrera GM. Pathogenesis of arrhythmias associated with metastatic tumors of the heart. N Engl J Med 1959; 260: 86971.

10. Samaras P, Stenner-Liewen F, Bauer S, et al. Images in cardiovascular medicine. Infarction-like electrocardiographic changes due to a myocardial metastasis from a primary lung cancer. Circulation 2007; 115: e320-1. 